UDC 517.28, 517.54, 517.41

V. V. Starkov

\title{
STRUCTURE OF KELLER MAPPINGS, TWO-DIMENSIONAL CASE
}

\begin{abstract}
A Keller map is a polynomial mapping $f: \mathbb{R}^{n} \rightarrow \mathbb{R}^{n}$ (or $\mathbb{C}^{n} \rightarrow \mathbb{C}^{n}$ ) with the Jacobian $J_{f} \equiv$ const $\neq 0$. The Jacobian conjecture was first formulated by O. N. Keller in 1939. In the modern form it supposes injectivity of a Keller map. Earlier, in the case $n=2$, the author gave a complete description of Keller maps with $\operatorname{deg} f \leq 3$. This paper is devoted to the description of Keller maps for which $\operatorname{deg} f \leq 4$. Significant differences between these two cases are revealed, which, in particular, indicate the irregular structure of Keller maps even in the case of $n=2$.
\end{abstract}

Key words: Jacobian conjecture, Keller maps

\section{Mathematical Subject Classification: $14 R 15$}

Let us denote the set of all polynomial mappings $f=\left(f_{1}, \ldots, f_{n}\right)$ : $\mathbb{R}^{n} \rightarrow \mathbb{R}^{n}$ (or $\mathbb{C}^{n} \rightarrow \mathbb{C}^{n}$ ) of degree $m \in \mathbb{N}$ by $P_{n}(m): f_{k}$ is a polynomial of $n$ variables of degree $\operatorname{deg} f_{k} \leq m$ for each $k=1, \ldots, n$. As usual, $D f$ is for the Jacobi matrix and $J_{f}$ for the Jacobian. The Jacobian Conjecture (JC) formulated by Keller [3] in 1939 in its modern form is:

If $f$ is a polynomial mapping and $J_{f} \equiv$ const $\neq 0$ then $f$ is injective in $\mathbb{R}^{n}\left(\mathbb{C}^{n}\right)$.

The proof of the conjecture would allow to use it widely in a number of branches of mathematics. Beside the one given above, other equivalent formulations also exist. Many publications are devoted to this conjecture: see, e.g., [1], [2], [4], [6], [11]. In particular, in [10] the conjecture is proved for $m=2$ for any $n$. In [5] it is checked for $f \in P_{2}(100)$. However, it has not been proved neither to be true nor to be false for the general case of any $n$. It is included in the list of "Mathematical Problems for the Next Century" [8]. 
A polynomial map $f$ is called a Keller map if $J_{f} \equiv$ const $\neq 0$. It is obvious that it is enough to prove the $\mathbf{J C}$ for the Keller mappings with $J_{f} \equiv 1$ and $D f(0)=I$ ( $I$ is for the identity matrix, $f(0)=0$ ). Therefore these properties are assumed in the sequel.

As the conjecture has neither been proved nor rejected for many years, it seems important to try to describe Keller mappings or their subclasses and then apply criteria or sufficient conditions of injectivity. Besides, Keller mappings for which the $\mathbf{J C}$ is true (see, e.g., [1]) are widely used in applications, which also make such study important.

In [9] Keller mappings were completely described for $n=2$ and $m=3$ : Theorem A. 9] Let $G \in P_{2}(3), G(0)=0, D G(0)=I$. Then $J_{G}(x, y) \equiv 1$ if and only if $G=A^{-1} \circ g \circ A$, where $g(x, y)=(U(x, y), V(x, y))$,

$U(x, y)=x+\alpha_{2}(x+y)^{2}+\alpha_{3}(x+y)^{3}, V(x, y)=y-\alpha_{2}(x+y)^{2}-\alpha_{3}(x+y)^{3}$,

$\alpha_{2}$ and $\alpha_{3}$ are arbitrary fixed constants, $A$ is a linear homogeneous nondegenerate mapping.

Injectivity of such mappings $G \in P_{2}(3)$ follows from injectivity of Keller mappings from $P_{2}(100)$ proved in [5]. Subclasses of Keller mappings where $\mathbf{J C}$ is true were described for any $n$ and $m$ in [7].

Theorem B. [7] Let $g(X)=\left(g_{1}, \ldots, g_{n}\right) \in P_{n}(m)$, where $X=\left(x_{1}, \ldots\right.$, $\left.x_{n}\right)$, and for $k=1, \ldots, n$

$$
\begin{gathered}
g_{k}(X)=x_{k}+\gamma_{k}\left[\alpha_{2}\left(x_{1}+\ldots+x_{n}\right)^{2}+\alpha_{3}\left(x_{1}+\ldots+x_{n}\right)^{3}+\ldots+\right. \\
\left.+\alpha_{m}\left(x_{1}+\ldots+x_{n}\right)^{m}\right]
\end{gathered}
$$

$\alpha_{j}, j=1, \ldots, m$, and $\gamma_{k}$ are arbitrary fixed constants with $\sum_{k=1}^{n} \gamma_{k}=0$. Then $g$ is a Keller map and $g$ is injective.

Note that for $n \geq 3$ (but not for $n=2$ !) [7] gave wider compared to theorem B subclasses of Keller mappings. Also note that in the case $n=2, m=3$ the mappings $g$ from Theorems $\mathrm{A}$ and $\mathrm{B}$ coincide. This gave birth to the conjecture (see [9]) that all Keller mappings in $P_{2}(m)$ have the form $G=A^{-1} \circ g \circ A$, where $g(x, y)=(u(x, y), v(x, y))$,

$$
u(x, y)=x+\sum_{k=2}^{m} \alpha_{k}(x+y)^{k}, \quad v(x, y)=y-\sum_{k=2}^{m} \alpha_{k}(x+y)^{k},
$$

$\alpha_{2}, \ldots, \alpha_{m}$ are arbitrary fixed constants, $A$ is from Theorem A. 
This conjecture is indirectly supported by Theorem C. With $X=(x, y) \in \mathbb{R}^{2}$, consider $f(X)=(u(X), v(X))$, where $u$ and $v$ are as in (1) and

$$
h(X)=(u(X)+w(X), v(X)+W(X)),
$$

where $W$ and $w$ are homogeneous polynomials of degree $(m+1)$ of $x$ and $y$. If $J_{h}(X) \equiv 1$, then $h=A^{-1} \circ F \circ A$, where $A$ is a linear homogeneous nondegenerate mapping and

$$
F(X)=\left(u(X)+\alpha_{m+1}(x+y)^{m+1}, v(X)-\alpha_{m+1}(x+y)^{m+1}\right),
$$

for some constant $\alpha_{m+1}$. The Jacobian conjecture is true for the mapping $h$.

This article is devoted to description of Keller mappings in $P_{2}(4)$. The structure of Keller mappings in $P_{2}(m)$ turned out to change when $m=2,3$ changes to $m=4$. $P_{2}(4)$ contains new Keller mappings beside those of form (1). The following main result is proved:

Theorem 1. The class of Keller mappings $f \in P_{2}(4)$ with $\operatorname{deg} f=4$ contains not only mappings $G$ of form (1) but also polynomial mappings $\Phi_{a, b, d}(x, y)=\left(\Phi_{1}, \Phi_{2}\right)$, where $a \neq 0 \neq b, d$ is any number, $d \neq \frac{a^{2}}{2 b}$,

$$
\begin{gathered}
\Phi_{1}=x+\frac{1}{2 a}(a x+b y)^{2}, \\
\Phi_{2}=y-\frac{1}{2 b}\left[(a x+b y)^{2}+x^{2}\left(2 b d-a^{2}\right)\right]+ \\
+x(a x+b y)^{2}\left(\frac{a}{2 b}-\frac{d}{a}\right)+\left(\frac{1}{8 b}-\frac{d}{4 a^{2}}\right)(a x+b y)^{4} .
\end{gathered}
$$

Also it contains mappings $F_{c, s}(x, y)=\left(F_{1}, F_{2}\right)$ with $c \neq 0 \neq s$,

$$
F_{1}=x+c y^{2}, \quad F_{2}=y-s x^{2}-2 c s x y^{2}-c^{2} s y^{4} .
$$

In particular, this theorem rejects the conjecture from [9] mentioned above about the structure of Keller mappings in $P_{2}(m)$. The case turnes out to be much more complicated.

Definition 1. Let $f, h \in P_{n}(m), f(0)=h(0)=0, D f(0)=D g(0)=I$. Mappings $f$ and $h$ are equivalent (denoted by $f \approx h$ ) if a linear nondegenerate mapping $A$ exists, such that $f=A^{-1} \circ h \circ A$. 
Theorem 2 below answers the question if equivalent mappings exist in classes $\left\{\Phi_{a, b, d}\right\}$ and $\left\{F_{c, s}\right\}$ and how many different (i.e., non-equivalent) mappings are there.

Theorem 2. Consider mappings $\left\{\Phi_{a, b, d}\right\}$ and $\left\{F_{c, s}\right\}$ defined in Theorem 1. Then for any $a \neq 0 \neq b, c \neq 0 \neq s$ and any $d \neq \frac{a^{2}}{2 b}$

$$
F_{c, s} \approx \Phi_{1,1,1}=\left(\begin{array}{l}
x+\frac{1}{2}(x+y)^{2} \\
y-\frac{1}{2}\left[(x+y)^{2}+x^{2}\right]-\frac{1}{2} x(x+y)^{2}-\frac{1}{8}(x+y)^{4}
\end{array}\right) \approx \Phi_{a, b, d} .
$$

Join Theorems 1 and 2 to see that beside familiar Keller mappings of form (1) $P_{2}(4)$ contains at least one non-equivalent to then mapping $\Phi_{1,1,1}$.

Remark. Proofs of Theorems 1 and 2 are valid both in the real and in the complex case, as well as Theorems $A$ and $B$.

Proof of Theorem 1. Let $f=\left(f_{1}, f_{2}\right) \in P_{2}(4)$ be a Keller mapping, $f(0)=0, \quad D f(0)=I, \operatorname{deg} f=4$. Then

$f_{1}=x+l(x, y)+l^{(2)}(x, y)+w(x, y), f_{2}=y+L(x, y)+L^{(2)}(x, y)+W(x, y)$,

where $l$ and $L$ are homogeneous polynomials of degree $2, l^{(2)}$ and $L^{(2)}$ of degree $3, w$ and $W$ of degree 4 . Compare the highest degrees in the identity $J_{f} \equiv 1$ to obtain $W_{x} w_{y}-w_{x} W_{y} \equiv 0$. This identity implies the equality

$$
w=\lambda W
$$

with some constant $\lambda$.

Identities $w \equiv 0$ and $W \equiv 0$ can not be valid simultaneously, because $\operatorname{deg} f=4$, equality (2) holds for $w \equiv 0$ and $W \not \equiv 0$ with $\lambda=0$ (in the symmetrical case $w \not \equiv 0$ and $W \equiv 0$ the similar equality $W=\delta w$ holds). Therefore, we can assume that $w \not \equiv 0$ and $W \not \equiv 0$.

Denote

$$
w(x, y)=\sum_{k=0}^{4} a_{k} x^{k} y^{4-k}=y^{4} \sum_{k=0}^{4} a_{k} t^{k}=y^{4} p(t)
$$




$$
W(x, y)=\sum_{k=0}^{4} b_{k} x^{k} y^{4-k}=y^{4} \sum_{k=0}^{4} b_{k} t^{k}=y^{4} q(t), \quad t=\frac{x}{y} .
$$

Then

$$
\begin{array}{cl}
w_{x}=y^{3} p^{\prime}(t), & w_{y}=y^{3}\left(4 p(t)-t p^{\prime}(t)\right), \\
W_{x}=y^{3} q^{\prime}(t), & W_{y}=y^{3}\left(4 q(t)-t q^{\prime}(t)\right) .
\end{array}
$$

Without loss of generality $w_{x} \not \equiv 0 \not \equiv W_{x}$; otherwise pass to the equivalent mapping $A^{-1} \circ f \circ A \approx f$ that satisfies these inequalities. Rewrite the equality $W_{x} w_{y}-w_{x} W_{y} \equiv 0$ in the form

$$
\frac{W_{y}}{W_{x}}=\frac{w_{y}}{w_{x}} \Longrightarrow \frac{4 q(t)-t q^{\prime}(t)}{q^{\prime}(t)}=\frac{4 p(t)-t p^{\prime}(t)}{p^{\prime}(t)} \Longrightarrow \frac{q^{\prime}(t)}{q(t)}=\frac{p^{\prime}(t)}{p(t)} .
$$

Therefore, $p(t)=\lambda q(t), \lambda=$ const, i.e., $w=\lambda W$.

Compare the fifth degree in equality $J_{f} \equiv 1$ to obtain the equation

$$
W_{y}\left(l^{(2)}-\lambda L^{(2)}\right)_{x}+W_{x}\left(\lambda L^{(2)}-l^{(2)}\right)_{y} \equiv 0 .
$$

It is obvious that this identity holds if $l^{(2)} \equiv \lambda L^{(2)}$. This is assumed further in this article leaving the other case $l^{(2)} \not \equiv \lambda L^{(2)}$ outside. So,

$$
f(X)=\left(\begin{array}{c}
x+l+\lambda L^{(2)}+\lambda W \\
y+L+L^{(2)}+W
\end{array}\right) .
$$

If $\lambda \neq 0$, pass from the mapping $f$ to the equivalent mapping

$$
F=A \circ f \circ A^{-1}=\left(\begin{array}{c}
x+\widetilde{l} \\
y+\widetilde{L}+\widetilde{L}^{(2)}+\widetilde{W}
\end{array}\right)
$$

with matrix $A=\left(\begin{array}{cc}1 & (-\lambda) \\ 0 & 1\end{array}\right)$. Here $\widetilde{l}$ and $\widetilde{L}$ are polynomials of degree 2 , $\operatorname{deg} \widetilde{L}^{(2)}=3, \operatorname{deg} \widetilde{W}=4$. Compare the fourth, third, second, and first degrees in the equality

$$
J_{F}=\left|\begin{array}{ll}
\left(1+\widetilde{l}_{x}\right) & \widetilde{l}_{y} \\
\left(\widetilde{L}_{x}+\widetilde{L}_{x}^{(2)}+\widetilde{W}_{x}\right) & \left(1+\widetilde{L}_{y}+\widetilde{L}_{y}^{(2)}+\widetilde{W}_{y}\right)
\end{array}\right| \equiv 1
$$

to get, respectively, the equations

$$
\widetilde{l}_{x} \widetilde{W}_{y}-\widetilde{l}_{y} \widetilde{W}_{x}=0
$$




$$
\begin{gathered}
\widetilde{W}_{y}+\widetilde{l}_{x} \widetilde{L}_{y}^{(2)}-\widetilde{l}_{y} \widetilde{L}_{x}^{(2)}=0, \\
\widetilde{L}_{y}^{(2)}+\widetilde{l}_{x} \widetilde{L}_{y}-\widetilde{l}_{y} \widetilde{L}_{x}=0 \\
\widetilde{L}_{y}+\widetilde{l}_{x}=0 .
\end{gathered}
$$

Rewrite $\widetilde{W}(x, y)$ as

$$
\widetilde{W}=y^{4} \widetilde{q}(t), \quad \widetilde{l} \approx y^{2} \sum_{k=0}^{2} c_{k} t^{k}=y^{2} r(t)
$$

similarly to $(3)$. Note that $\widetilde{l} \not \equiv 0$; otherwise equality $J_{F} \equiv 1$ yields $0=$ $=\widetilde{L}_{y}=\widetilde{L}_{y}^{(2)}=\widetilde{W}_{y}$, i.e., $\widetilde{L}, \widetilde{L}^{(2)}, \widetilde{W}$ depend only on $x$. Pass from $F$ to the equivalent mapping $F_{1}=A^{-1} \circ F \circ A$ with matrix $A=\left(\begin{array}{ll}1 & 1 \\ 0 & 1\end{array}\right)$ and check that $F_{1}$ coincides with $g$ from $(1)$. So, the case $\widetilde{l} \equiv 0$ gives no new Keller mappings beside those listed in (1).

Only one of the two possibilities holds: either $\widetilde{l}_{x} \not \equiv 0 \not \equiv \widetilde{W}_{x}$ or $\widetilde{l}_{y} \not \equiv 0 \not \equiv$ $\not \equiv \widetilde{W}_{y}$. Otherwise if $\widetilde{l}_{x} \equiv 0$ then (4) and the condition $\widetilde{l} \not \equiv 0$ imply $\widetilde{W}_{x} \equiv 0$. But then the symmetrical condition $\widetilde{l}_{y} \not \equiv 0 \not \equiv \widetilde{W}_{y}$ holds because $W \not \equiv 0$. So in the sequel $\widetilde{l}_{x} \not \equiv 0 \not \widetilde{W}_{x}$ can be assumed to hold. Rewrite (4) in the form $\frac{\widetilde{W}_{y}}{\widetilde{W}_{x}}=\frac{\widetilde{l}_{y}}{\widetilde{l}_{x}}$. Then $\frac{4 \widetilde{q}}{\widetilde{q}^{\prime}}=\frac{2 r}{r^{\prime}} \Rightarrow \widetilde{q}=\mu r^{2}(t), \mu=$ const, follows in the same way as in consideration of the equation $W_{x} w_{y}-w_{x} W_{y}=0$; then

$$
\widetilde{W}(x, y)=y^{4} \widetilde{q}(t)=\mu \widetilde{l}^{2}(x, y), \quad \widetilde{W}_{x}=2 \mu \widetilde{l}_{x} \widetilde{l}, \quad \widetilde{W}_{y}=2 \mu \widetilde{l}_{y} \widetilde{l} .
$$

Now (5) can be rewritten as

$$
\widetilde{l}_{y}\left(2 \mu \widetilde{l}-\widetilde{L}_{x}^{(2)}\right)+\widetilde{l}_{x} L_{y}^{(2)}=0 .
$$

Denote $\widetilde{l}_{x}=a x+b y$; then $\tilde{l}=\frac{a}{2} x^{2}+b x y+c y^{2}$ (here $a, b, c$ are constants). This together with (7) implies

$$
\widetilde{L}=-a x y-\frac{b}{2} y^{2}-d x^{2}, d=\text { const. }
$$

From $(6)$ derive $\widetilde{L}_{y}^{(2)}=\left(\widetilde{l}_{x}\right)^{2}+\widetilde{l}_{y} \widetilde{L}_{x}=(a x+b y)^{2}-(b x+2 c y)(a y+2 d x)$. 
First consider the case $b \neq 0$, when

$$
\widetilde{L}^{(2)}=\frac{1}{3 b}(a x+b y)^{3}-\left[\frac{2 a c}{3} y^{3}+2 b d x^{2} y+\left(\frac{a b}{2}+2 c d\right) x y^{2}\right]+u x^{3},
$$

$u=$ const. Taking this last equality into account, rewrite (8) in the form

$$
\begin{gathered}
(b x+2 c y)\left[2 \mu\left(\frac{a}{2} x^{2}+b x y+c y^{2}\right)-\frac{a}{b}(a x+b y)^{2}+4 b d x y+\right. \\
\left.+\left(\frac{a b}{2}+2 c d\right) y^{2}-3 u x^{2}\right]+(a x+b y)\left[(a x+b y)^{2}-(b x+2 c y)(a y+2 d x)\right]=0 \Longleftrightarrow \\
\Longleftrightarrow(b x+2 c y)\left[2 \mu\left(\frac{a}{2} x^{2}+b x y+c y^{2}\right)-\frac{a}{b}(a x+b y)^{2}+4 b d x y+\right. \\
\left.+\left(\frac{a b}{2}+2 c d\right) y^{2}-(a x+b y)(a y+2 d x)-3 u x^{2}\right]+(a x+b y)^{3}=0
\end{gathered}
$$

Compare coefficients at equal powders in (9) to obtain the following equations:

$$
\begin{gathered}
x^{3}: \quad \mu a b-2 a b d-3 n u=0, \\
x^{2} y: \quad 2 \mu b^{2}+2 a c \mu-2 a^{3} \frac{c}{b}+2 b^{2} d-4 c a d-6 c u=0, \\
x y^{2}: \quad 6 \mu b e+\frac{3}{2} a b^{2}-6 a^{2} c+6 b c d=0, \\
y^{3}: \quad 4 c^{2} \mu-3 a b c+4 c^{2} d+b^{3}=0 .
\end{gathered}
$$

From (10) we determine

$$
3 u=a(\mu-2 d)
$$

Substitute (14) into (11) to get $\mu=\frac{a^{3}}{b^{3}} c-d$.

Now (12) and (13) can be rewritten as the system of equations

$$
\left\{\begin{array}{l}
a b^{4}+4 a^{3} c^{2}-4 a^{2} b^{2} c=0 \\
4 a^{3} c^{3}+b^{6}-3 a b^{4} c=0
\end{array}\right.
$$

Substract the first equation from the second one multiplied on $c$ to obtain

$$
\left\{\begin{array}{l}
a\left(b^{4}-4 a c b^{2}+4 a^{2} c^{2}\right)=0 \\
b^{2}\left(b^{4}-4 a c b^{2}+4 a^{2} c^{2}\right)=0
\end{array}\right.
$$


i.e., $b^{2}=2 a c$. So, $a \neq 0$ and $c=\frac{b^{2}}{2 a}$.

Now we see that in the considered case the Jacobian $J_{F} \equiv 1$ if and only if for any $d$

$$
a \neq 0 \neq b, \quad c=\frac{b^{2}}{2 a}, \quad \mu=\frac{a^{3} c}{b^{3}}-d=\frac{a^{2}}{2 b}-d, \quad 3 u=a\left(\frac{a^{2}}{2 b}-3 d\right) .
$$

So

$$
\begin{gathered}
\widetilde{l}=\frac{a}{2} x^{2}+b x y+\frac{b^{2}}{2 a} y^{2}=\frac{1}{2 a}(a x+b y)^{2}, \\
\widetilde{L}=-\left(d x^{2}+a x y+\frac{b}{2} y^{2}\right)=-\frac{1}{2 b}\left[(a x+b y)^{2}+x^{2}\left(2 b d-a^{2}\right)\right], \\
\widetilde{L}^{(2)}=\frac{1}{3 b}(a x+b y)^{3}-\left[\frac{2 a c}{3} y^{3}+2 b d x^{2} y+\left(\frac{a b}{2}+2 c d\right) x y^{2}\right]+u x^{3}= \\
=\frac{1}{3 b}(a x+b y)^{3}-\left[\frac{b^{2}}{3} y^{3}+2 b d x^{2} y+\left(\frac{a b}{2}+\frac{d b^{2}}{a}\right) x y^{2}\right]+\left(\frac{a^{3}}{6 b}-a d\right) x^{3}= \\
=\frac{1}{3 b}(a x+b y)^{3}-\frac{b^{2}}{3} y^{3}-\frac{a b}{2} x y^{2}+\frac{a^{3}}{6 b} x^{3}-d x\left(2 b x y+\frac{b^{2}}{a} y^{2}+a x^{2}\right)= \\
=\frac{a^{3}}{2 b} x^{3}+a^{2} x^{2} y+\frac{a b x y^{2}}{2}-\frac{d x}{a}(a x+b y)^{2}=(a x+b y)^{2} x\left(\frac{a}{2 b}-\frac{d}{a}\right) . \\
\widetilde{W}=\mu(\widetilde{l})^{2}=\left(\frac{1}{8 b}-\frac{d}{4 a^{2}}\right)(a x+b y)^{4} .
\end{gathered}
$$

Let us show that the polynomial mapping

$$
F=\Phi_{a, b, d}=\left(\begin{array}{l}
x+\widetilde{l} \\
y+\widetilde{L}+\widetilde{L}^{(2)}+\widetilde{W}
\end{array}\right)
$$

is not equivalent to the mapping $g$ from (1) for all $a, b, d(a \neq 0 \neq b)$.

Assume that this is not true, so a mapping $g$ and a matrix $A$ from (17) exist, such that

$$
g(A X)=A\left(\Phi_{a, b, d}(X)\right)
$$

For the polynomial mapping $f$ of $\operatorname{deg} f=m$ denote

$$
f=\sum_{k-0}^{m}[f]_{k}
$$


where $[f]_{k}$ are homogeneous mappings of degree $k$. Let the matrix

$$
A=\left(\begin{array}{ll}
\alpha & \beta \\
\gamma & \delta
\end{array}\right), \quad\left(\begin{array}{l}
x_{1} \\
y_{1}
\end{array}\right):=A X=\left(\begin{array}{c}
\alpha x+\beta y \\
\gamma x+\delta y
\end{array}\right), \quad \operatorname{det} A \neq 0 .
$$

$z_{1}:=x_{1}+y_{1}$. Then (16) implies $A\left[\Phi_{a, b, d}(X)\right]_{4}=[g(A X)]_{4}$, i.e.,

$$
\begin{gathered}
A\left(\begin{array}{l}
0 \\
\left(\frac{1}{8 b}-\frac{d}{4 a^{2}}\right)(a x+b y)^{4}
\end{array}\right)=\left(\begin{array}{c}
\beta\left(\frac{4}{8 b}-\frac{d}{4 a^{2}}\right)(a x+b y)^{4} \\
\delta\left(\frac{1}{8 b}-\frac{d}{4 a^{2}}\right)(a x+b y)^{4}
\end{array}\right)= \\
=\alpha_{4} z_{1}^{4}\left(\begin{array}{c}
1 \\
-1
\end{array}\right),
\end{gathered}
$$

$\alpha_{4}$ is the constant from (1). Then

$$
\delta=-\beta, \quad a x+b y=\eta(\alpha+\gamma) x, \quad \eta=\mathrm{const} ;
$$

this is possible only if $b=0$ which contradicts the current assumption $b \neq 0$. So, the mappings $\Phi_{a, b, d}$ with parameters defined in (15) are new polynomial mappings with $J_{F} \equiv 1$, not equivalent to the mappings $G=$ $=A^{-1} \circ g \circ A$ from (1).

Now we need to consider the case $b=0$. Then $\widetilde{L}^{(2)}$ has the form

$$
\widetilde{L}^{(2)}=a^{2} x^{2} y-\left(\frac{2 a c}{3} y^{3}+2 c d x y^{2}\right)+u x^{3}
$$

and (8) can be written down as

$2 c y\left[2 \mu\left(\frac{a}{2} x^{2}+c y^{2}\right)-2 a^{2} x y+2 c d y^{2}-a x(a y+2 d x)-3 u x^{2}\right]+(a x)^{3}=0$.

Equation $a=0$ corresponds to equation (10).

Keeping this in mind, rewrite (18):

$$
2 c y\left[2 \mu c y^{2}+2 c d y^{2}-3 u x^{2}\right]=0 .
$$

This equality is equivalent to one of the two conditions holding:

i) $c=0$ or ii) $\left\{\begin{array}{l}2 c(\mu+d)=0 \\ 3 u=0\end{array}\right.$

i) Let $c=0$. Then $\widetilde{l} \equiv 0$ and $\widetilde{W} \equiv 0$; this contradicts the assumptions of Theorem 1: $\operatorname{deg} f=4$. So, $c \neq 0$. 
ii) In this case $a=b=0=u, \quad c \neq 0, \quad \mu=-d$; then $\widetilde{l}=c y^{2}$, $\widetilde{L}=-d x^{2}, \widetilde{L}^{(2)}=-2 c d x y^{2}, \widetilde{W}=-d c^{2} y^{4}$ with $d \neq 0$, otherwise $\widetilde{W}=0$.

Therefore,

$$
F=F_{c, d}=\left(\begin{array}{l}
x+c y^{2} \\
y-d x^{2}-2 c d x y^{2}-d c^{2} y^{4}
\end{array}\right), \quad c \neq 0 \neq d .
$$

Let us show that the mappings $F_{c, d}$ and $g$ from (1) are significantly different, i.e., $g \not \approx F_{c, d}$. To do this assume the contrary and consider the equality $\left[A^{-1} \circ F_{c, d} \circ A\right]_{4}=[g]_{4}$ using notation (17):

$$
A^{-1}\left(\begin{array}{l}
0 \\
-d c^{2} y_{1}^{4}
\end{array}\right)=\left(\begin{array}{l}
\alpha_{4} \\
-\alpha_{4}
\end{array}\right)(x+y)^{4}
$$

This implies $(\gamma x+\delta y)=\eta(x+y), \eta=$ const, i.e., $\gamma=\delta=\eta$. Then

$$
\left(\begin{array}{l}
0 \\
-d c^{2} \eta^{4}(x+y)^{4}
\end{array}\right)=\left(\begin{array}{ll}
\alpha & \beta \\
\gamma & \gamma
\end{array}\right)\left(\begin{array}{l}
1 \\
-1
\end{array}\right) \alpha_{4}(x+y)^{4}
$$

Finally $d c^{2}=0$ and we have got a contradiction with the condition $c \neq 0 \neq d$. Therefore, $g \not \approx F_{c, d}$. Theorem 1 is proved.

Proof of Theorem 2. Show that $F_{c, s} \approx \Phi_{1,1,1}$ for any $c \neq 0 \neq s$, i.e., a matrix $A$ (see $(17))$ exists, such that $A \Phi_{1,1,1}(X)=F_{c, s}(A X)$.

$$
\begin{gathered}
A \Phi_{1,1,1}(X)=A X+A\left(\begin{array}{l}
\frac{1}{2}(x+y)^{2}-1 \\
-\frac{1}{2}\left[(x+y)^{2}+x^{2}\right]-\frac{1}{2}(x+y)^{2} x-\frac{1}{8}(x+y)^{4}
\end{array}\right) ; \\
F_{c, s}(A X)=A X+ \\
+\left(\begin{array}{c}
c(\gamma x+\delta y)^{2} \\
-s(\alpha x+\beta y)^{2}-2 c s(\alpha x+\beta y)(\gamma x+\delta y)^{2}-c^{2} s(\gamma x+\delta y)^{4}
\end{array}\right) .
\end{gathered}
$$

Compare the first coordinates in the equality $\left[A \Phi_{1,1,1}(X)\right]_{4}=\left[F_{c, s}(A X)\right]_{4}$ to obtain $\beta\left(-\frac{1}{8}\right)(x+y)^{4}=0$, i.e., $\beta=0$. Comparing the second coordinates yields

$$
-\frac{1}{8} \delta(x+y)^{4}=-c^{2} s(\gamma x+\delta y)^{4} \quad \Longrightarrow \quad \gamma=\delta=8 c^{2} s \delta^{4}, \quad \delta=\frac{1}{2 \sqrt[3]{c^{2} s}} .
$$


Comparing the first coordinates of the identity $\left[A \Phi_{1,1,1}(X)\right]_{2}=\left[F_{c, s}(A X)\right]_{2}$ obtain $\alpha=2 c \delta^{2}$. The second coordinates give the relation

$$
\delta=2 s \alpha^{2}=2 s \cdot 4 c^{2} \delta^{4} \Longleftrightarrow 1=8 c^{2} s \delta^{3}
$$

we already have. The same equality appears if we consider the identity $\left[A \Phi_{1,1,1}(X)\right]_{3}=\left[F_{c, s}(A X)\right]_{3}$ :

$$
-\frac{\delta}{2}(x+y)^{2} x=-2 \operatorname{cs} \alpha \delta^{2} x(x+y)^{2} \Longleftrightarrow \delta=4 \operatorname{cs} \alpha \delta^{2}=8 c^{2} s \delta^{4} .
$$

So, for any $c \neq 0 \neq s$ a matrix $A$ from (17) exists and

$$
\beta=0, \quad \gamma=\delta=\frac{1}{2 \sqrt[3]{c^{2} s}}, \quad \alpha=2 c \delta^{2}=\frac{1}{2 \sqrt[3]{c s^{2}}},
$$

i.e., $F_{c, s} \approx \Phi_{1,1,1}$.

To complete the proof of Theorem 2 it is enough now to show that $\Phi_{1,1,1} \approx \Phi_{a, b, d}$ for any $a \neq 0 \neq b, \quad d \neq \frac{a^{2}}{2 b}$ : we need to find a matrix $B$ with $\operatorname{det} B \neq 0$ such that $\Phi_{1,1,1}(B X)=B \Phi_{a, b, c}(X)$. Let us show that the matrix

$$
B=\left(\begin{array}{ll}
4 a \delta^{2} & 0 \\
2 \delta a(1-2 \delta) & 2 b \delta
\end{array}\right), \quad \delta=\sqrt[3]{\frac{b d}{4 a^{2}}-\frac{1}{8}}
$$

satisfies this condition. The condition of equivalence of the mappings implies $\delta \neq 0$. Evaluate

$$
\begin{gathered}
\Phi_{1,1,1}(B X)=B X+\left(\begin{array}{l}
2 \delta^{2}(a x+b y)^{2} \\
-2 \delta^{2}(a x+b y)^{2}-8 a^{2} \delta^{4} x^{2}
\end{array}\right)+ \\
+\left(\begin{array}{l}
0 \\
-8 \delta^{4} a x(a x+b y)^{2}-2 \delta^{4}(a x+b y)^{4}
\end{array}\right) \\
B \Phi_{a, b, d}(X)=B X+B\left(\begin{array}{l}
\frac{1}{2 a}(a x+b y)^{2} \\
-\frac{1}{2 b}\left[(a x+b y)^{2}+\left(2 b d-a^{2}\right) x^{2}\right]
\end{array}\right)+ \\
+B\left(\begin{array}{l}
0 \\
x(a x+b y)^{2}\left(\frac{a}{2 b}-\frac{d}{a}\right)+\left(\frac{1}{8 b}-\frac{d}{4 a^{2}}\right)(a x+b y)^{4}
\end{array}\right)
\end{gathered}
$$


The condition $\left[\Phi_{1,1,1}(B X)\right]_{4}=\left[B \Phi_{a, b, d}(X)\right]_{4}$ is equivalent to the equality

$$
-2 \delta^{4}=2 b \delta\left(\frac{1}{8 b}-\frac{d}{4 a^{2}}\right) \Longleftrightarrow \delta^{3}=\frac{b d}{4 a^{2}}-\frac{1}{8} .
$$

Compare the first coordinates in the condition

$$
\left[\Phi_{1,1,1}(B X)\right]_{2}=\left[B \Phi_{a, b, d}(X)\right]_{2}
$$

to get the obvious equality $2 \delta^{2}=\frac{4 a \delta^{2}}{2 a}$; the second coordinates give another obvious equality

$$
\begin{aligned}
-2 \delta^{2}(a x+b y)^{2}-8 a^{2} \delta^{4} x^{2} & =\delta(1-2 \delta)(a x+b y)^{2}-\delta\left[(a x+b y)^{2}+\right. \\
\left.+\left(2 b d-a^{2}\right) x^{2}\right] & \Longleftrightarrow 8 a^{2} \delta^{3}=\left(2 b d-a^{2}\right) .
\end{aligned}
$$

To complete the proof, we need now only to check the identity

$$
\left[\Phi_{1,1,1}(B X)\right]_{3}=\left[B \Phi_{a, b, d}(X)\right]_{3}
$$

for the second coordinates:

$$
-8 a \delta^{4} x(a x+b y)^{2}=2 b \delta\left(\frac{a}{2 b}-\frac{d}{a}\right) x(a x+b y)^{2} \Longleftrightarrow \delta^{3}=\frac{d b}{4 a^{2}}-\frac{1}{8} .
$$

Therefore, for any $a \neq 0 \neq b, \quad d \neq \frac{a^{2}}{2 b}, \quad c \neq 0 \neq s$ the relation $\Phi_{a, b, d} \approx \Phi_{1,1,1} \approx F_{c, s}$ holds. Theorem 2 is proved.

The next statement declares existence of equivalent Keller mappings $g$ of form (1): a subclass of $P_{2}(4)$ of different (pairwise non-equivalent) mappings of form (1) is constructed.

Proposition. Any mapping $g=g_{\alpha_{2}, \alpha_{3}, \alpha_{4}} \in P_{2}(4)$ of form (1) with $\alpha_{2} \neq 0 \neq \alpha_{3}$ defines a constant $s=\frac{\alpha_{4} \alpha_{2}}{\alpha_{3}^{2}}$ such that $g_{\alpha_{2}, \alpha_{3}, \alpha_{4}} \approx g_{1,1, s}$.

Proof. Choose arbitrary constants $\alpha_{2} \neq 0, \alpha_{3} \neq 0$, and $\alpha_{4}$ in the mapping $g=g_{\alpha_{2}, \alpha_{3}, \alpha_{4}} \in P_{2}(4)$. Let us prove that a constant $s$ and a matrix $A$ from (17) exist, such that

$$
g_{\alpha_{2}, \alpha_{3}, \alpha_{4}}(A X)=A g_{1,1, s}(X),
$$

i.e.,

$$
g_{\alpha_{2}, \alpha_{3}, \alpha_{4}}(A X)=A X+\left(\alpha_{2}[(\alpha+\gamma) x+(\beta+\delta) y]^{2}+\right.
$$




$$
\begin{aligned}
& \left.+\alpha_{3}[(\alpha+\gamma) x+(\beta+\delta) y]^{3}+\alpha_{4}[(\alpha+\gamma) x+(\beta+\delta) y]^{4}\right)\left(\begin{array}{c}
1 \\
-1
\end{array}\right)= \\
& =A g_{1,1, s}(X)=A X+\left[(x+y)^{2}+(x+y)^{3}+s(x+y)^{4}\right]\left(\begin{array}{c}
\alpha-\beta \\
\gamma-\delta
\end{array}\right) .
\end{aligned}
$$

Compare coefficients in this equality to get the folowing equations:

$$
\begin{gathered}
\alpha-\beta=-(\gamma-\delta) \Longleftrightarrow \alpha+\gamma=\beta+\delta \\
\alpha_{2}(\alpha+\gamma)^{2}=\alpha-\beta, \quad \alpha_{3}(\alpha+\gamma)^{3}=(\alpha-\beta), \quad \alpha_{4}(\alpha+\gamma)^{4}=s(\alpha-\beta) .
\end{gathered}
$$

Denote $\lambda=\alpha+\gamma=\beta+\delta$ and rewrite these equations in the form $\alpha_{2} \lambda^{2}=\alpha-\beta, \quad \alpha_{3} \lambda^{3}=\alpha-\beta, \quad \alpha_{4} \lambda^{4}=s(\alpha-\beta)$. Then the values of $\lambda$ and $s$ can be determined:

$$
\lambda=\alpha_{2} / \alpha_{3} \neq 0, \quad s=\frac{\alpha_{4} \lambda^{4}}{\alpha-\beta}=\frac{\alpha_{4} \lambda^{4}}{\alpha_{2} \lambda^{2}}=\frac{\alpha_{4} \lambda^{2}}{\alpha_{2}}=\frac{\alpha_{4} \alpha_{2}}{\alpha_{3}^{2}} .
$$

The elements of the matrix $A$ are constructed as follows: choose any $\alpha$ and $\gamma=\lambda-\alpha, \beta=\alpha-\alpha_{2} \lambda^{2}, \delta=\lambda-\beta=\lambda-\alpha+\alpha_{2} \lambda^{2}$. Check that $\operatorname{det} A \neq 0$ :

$$
\operatorname{det} A=\alpha \delta-\beta \gamma=\alpha\left(\lambda-\alpha+\alpha_{2} \lambda^{2}\right)-\left(\alpha-\alpha_{2} \lambda^{2}\right)(\lambda-\alpha)=\lambda^{3} \alpha_{2} \neq 0 .
$$

The proposition is proved.

Remark. Note that in the Proposition the constant $s$ significantly depends on the chosen $\alpha_{2}, \alpha_{3}, \alpha_{4}$.

Acknowledgment. The work is supported by the Russian Science Foundation under grant 17-11-01229 and performed in Petrozavodsk State University.

\section{References}

[1] Drużkowski L. M. On the global asymptotic stability problem and the Jacobian conjecture. Control and Cybernetics, 2005, vol. 34, no. 3, pp. 747-762.

[2] van den Essen A. Polynomial Automorphisms and the Jacobian Conjecture. Volume 190 of Progress in Mathematics, Birkhäuser Verlag, Basel, 2000.

[3] Keller O.-H. Ganze Cremona-Transformationen. Monatshefte Math. Phys., 1939, vol. 47, pp. 299-306. DOI: 10.1007/BF01695502. 
[4] Kulikov V. S. Generalized and local Jacobian problems. Russian Academy of Sciences. Izvestiya Mathematics, 1993, vol. 41, no. 2, pp. 351-365. DOI: 10.1070/IM1993v041n02ABEH002265.

[5] Moh T. T. On the global Jacobian conjecture and the configuration of roots. J. reine und angew. Math., 1983, vol. 340, pp. 140-212.

[6] Pinchuk S. A counterexample to the strong real Jacobian conjecture, Math. Z., 1994, vol. 217, pp. 1-4. DOI: 10.1007/BF02571929.

[7] Ponnusamy S. and Starkov V. V. The Jacobian conjecture and injectivity conditions. arXiv:1705.10921 [math.CV].

[8] Smale S. Mathematical Problems for the Next Century. Math. Intelligencer, 1998, vol. 20, no. 2, pp. 7-15. DOI: 10.1007/BF03025291.

[9] Starkov V. V. Jacobian conjecture, two-dimensional case. Probl. Anal. Issues Anal., 2016, vol. 5 (23), no. 2, pp. 69-78. DOI: 10.15393/j3.art.2016.3510.

[10] Wang S. S.-S. A Jacobian criterion for separability. J. of Algebra, 1980, vol. 65, no. 2, pp. 453-494. DOI: 10.1016/0021-8693(80)90233-1.

[11] Yagzhev A. V. Keller's problem. Siberian Math. J., 1980, vol. 21, no. 5, pp. 747-754. DOI: 10.1007/BF00973892.

Received May 24, 2017.

In revised form, June 8, $201 \%$.

Accepted June 8, 2017.

Published online June 16, 2017.

Petrozavodsk State University

33, Lenina pr., Petrozavodsk 185910, Russia

E-mail: vstar@petrsu.ru 